\title{
A STATIC ANALYSIS OF NONUNIFORM COLUMN BY STOCHASTIC FINITE ELEMENT METHOD USING WEIGHTED INTEGRATION APPROACH
}

\author{
Ta Duy Hien ${ }^{1,2}$ \\ ${ }^{1}$ University of Transport and Communications, No 3 Cau Giay Street, Hanoi, Vietnam \\ ${ }^{2}$ Research and Application center for technology in Civil Engineering (RACE), University of \\ Transport and Communications, No 3 Cau Giay Street, Hanoi, Vietnam \\ ARTICLE INFO \\ TYPE: Research Article \\ Received: 19/2/2020 \\ Revised: 29/3/2020 \\ Accepted: 10/4/2020 \\ Published online: 28/5/2020 \\ https://doi.org/10.25073/tcsj.71.4.5 \\ * Corresponding author \\ Email: tdhien@utc.edu.vn

\begin{abstract}
In general, the fluctuation of the elastic modulus of materials is crucial in structural analysis. This paper develops a stochastic finite element method (SFEM) for analyzing a nonuniform column considering the random process in elastic modulus. This random process of elastic modulus is assumed as a one-dimensional Gaussian random field. The weighted integration method is used to discretize the random field and establish the stochastic finite element formulation to compute the first and second moments of displacement fields. The results of the proposed approach are validated with those of the previous study. The response variability of displacement of column and effect of the parameter of the random field is investigated in detail.
\end{abstract}

Keywords: Nonuniform column, weighed integration method, SFEM, random field

C) 2020 University of Transport and Communications

\section{INTRODUCTION}

All materials in engineering have inherent uncertainties due to variables quality and inaccuracy of fabrication technology, manufacturing techniques. Normally, deterministic analysis $[1,2]$ is insufficient to provide complete information about the structural response. Thus, the deterministic analysis of structures needs to be complemented with the theory of random processes and fields to encompass the uncertain behaviors in the structural responses, i.e., the response variability. 
In recent years, the stochastic finite element method has been a topic of active research [3-6]. For finite element implementation, it is necessary to discretize such fields into random vector representations. Various methods developed for the discretization of random fields such as Karhunen-Loève expansion [7], nodal point method [8], midpoint method [9], the integration point method [8], a local averaging method [10], a weighted integral method [11, 12]. Hyuk Chun Noh [13] developed an SFEM using the weighted integral approach to determine the response variability of in-plane and plate structures with multiple uncertain elastic moduli and Poisson's ratio. T.D. Hien et al. [14] computed the variability of displacements of a beam subjected to a moving load with various random parameters by Monte Carlo simulation. Kitipornchai et al. [15] used the first-order perturbation technique incorporating mixed type and semi-analytical approach to derive the standard eigenvalue problem the functionally graded laminates beam based on the third-order shear deformation theory.

Besides the stochastic finite element method, there are limited studies on problems with stochasticity which have used other methods such as meshfree method, isogeometric analysis. Rahman et al. [16] developed a stochastic meshless method based on the element-free Galerkin method for in linear elasticity considering a homogeneous random field. N.X Hoang et al. [17] and T.D. Hien et al. [18] developed stochastic isogeometric analysis for the eigenvalue problem of composite structures with uncertain material properties. Chensen et al. [19] proposed the isogeometric generalized n-th order perturbation-based stochastic method for composite structures with random material parameters. Larrard et al. [20] studied the effect of the elastic modulus variability on the mechanical behavior of a nuclear containment vessel.

The paper is organized as follows. In Section 2, the finite element formulation for a nonuniform column with uncertain elastic modulus is developed using a weighed integration technique for discretization random field. Section 3 employs a numerical example and discussion. Section 4 accomplishes the conclusions.

\section{STOCHASTIC FINITE ELEMENT FORMULATION FOR NONUNIFORM COLUMN}

We consider a non-uniform column with a random property of elastic modulus as shown in Figure 1:

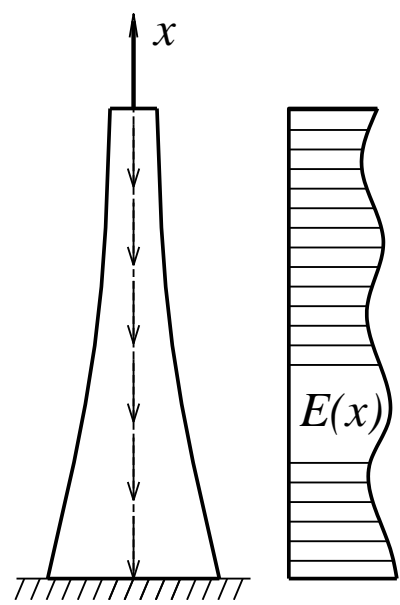

Figure 1. Model of a nonuniform column with uncertain elastic modulus E. 


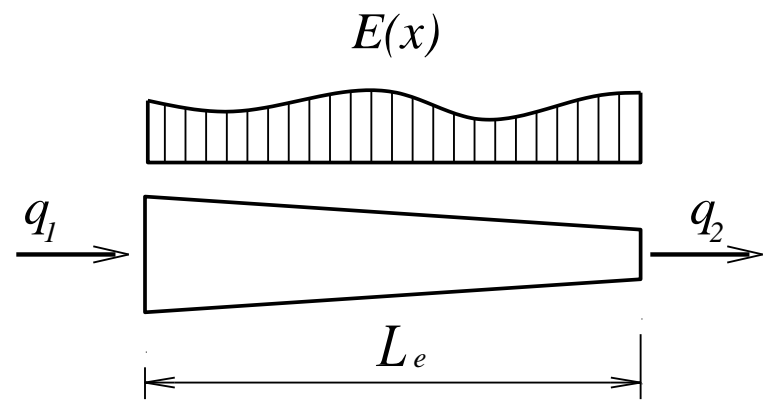

Figure 2. Bar finite element with a nonuniform cross-section and random process in elastic modulus.

For the non-uniform column, the bar element with the two-degree of freedoms is suitable for the column as shown in Fig. 2. The displacement $u(x)$ is interpolated by Lagrange function as follows:

$$
u(x)=\left[\begin{array}{ll}
N_{1} & N_{2}
\end{array}\right]\{q\}_{e}=[N]\{q\}_{e}
$$

where $N_{1}, N_{2}$ are Hamite function:

$$
\left\{\begin{array}{l}
N_{1}=1-\frac{x}{L_{e}} \\
N_{2}=\frac{x}{L_{e}}
\end{array}\right.
$$

and displacement vector of the element:

$$
\{q\}_{e}=\left\{\begin{array}{l}
q_{1} \\
q_{2}
\end{array}\right\}
$$

We assume that the cross-section of the column is linear variability as follows:

$$
A_{e}(x)=A_{1 e}\left(1-\frac{x}{L_{e}}\right)+A_{2 e} \frac{x}{L_{e}}
$$

In this study, the random process of elastic modulus $E(x)$ is assumed as a Gaussian random filed. The first statistical moments (mean), autocorrelation function and autocovariance function of a random process $E(x)$ are defined by:

$$
\begin{aligned}
& \bar{E}[E(x)]=E_{0}=\int_{-\infty}^{\infty} E(x) p\{E(x)\} d E(x) \\
& R(\tau)=\bar{E}[E(x) E(x+\tau)]=\int_{-\infty}^{\infty} \int_{-\infty}^{\infty} E(x) E(x+\tau) d E(x) d E(x+\tau) \\
& K(\tau)=\operatorname{Cov}[E(x) E(x+\tau)]=\int_{-\infty}^{\infty} \int_{-\infty}^{\infty}\left\{E(x)-E_{0}\right\}\left\{E(x+\tau)-E_{0}\right\} d E(x) d E(x+\tau)
\end{aligned}
$$

The power spectral density (or power spectrum) of random process $E(x)$ is defined as the Fourier transform of $R(\tau)$ : 
Transport and Communications Science Journal, Vol. 71, Issue 4 (05/2020), 359-367

$$
S(\omega)=\int_{-\infty}^{\infty} R(\tau) e^{-j \omega \tau} d \tau
$$

The random process of elastic modulus $E(x)$ is represented as follows:

$$
E(x)=E_{0}[1+r(x)]
$$

where $r(x)$ is a one-dimensional Gaussian random field with a mean equal to zero. We compute the stiffness matrix includes a random process of elastic modulus:

$$
\begin{aligned}
& {[K]_{e}=\int_{V_{e}}[B]^{T}[D][B] d V} \\
& =\int_{0}^{L_{e}}\left[\begin{array}{c}
-\frac{1}{L_{e}} \\
\frac{1}{L_{e}}
\end{array}\right] E_{0}(1+r(x))\left\{A_{1 e}\left(1-\frac{x}{L_{e}}\right)+A_{2 e} \frac{x}{L_{e}}\right\}\left[\begin{array}{ll}
-\frac{1}{L_{e}} & \frac{1}{L_{e}}
\end{array}\right] d x \\
& =\frac{E_{0}\left(A_{1 e}+A_{2 e}\right)}{2 L_{e}}\left[\begin{array}{cc}
1 & -1 \\
-1 & 1
\end{array}\right]+\frac{E_{0} A_{1 e}}{L_{e}}\left[\begin{array}{cc}
1 & -1 \\
-1 & 1
\end{array}\right] \int_{0}^{L_{e}} r(x) d x+ \\
& \frac{E_{0} A_{1 e}}{L_{e}}\left[\begin{array}{cc}
1 & -1 \\
-1 & 1
\end{array}\right]_{0}^{L_{e}} x\left(A_{2 e}-A_{1 e}\right) r(x) d x \\
& =[K]_{e}^{0}+[C]_{e}^{1} R_{1}^{e}+[C]_{e}^{2} R_{2}^{e}
\end{aligned}
$$

Random variables $R_{1}^{e}, R_{2}^{e}$ are represented by the integration of random process:

$$
R_{1}^{e}=\int_{0}^{L_{e}} r(x) d x ; \quad R_{2}^{e}=\int_{0}^{L_{e}} x\left(A_{2 e}-A_{1 e}\right) r(x) d x
$$

Stiffness matrix and displacement vector are expanded by Taylor's series:

$$
\begin{aligned}
& {\left[K\left(R_{1}^{e}, R_{2}^{e}, \ldots\right)\right]=[K]_{0}+\sum_{e=1}^{N e} \sum_{i=1}^{N r} \frac{\partial[K]}{\partial R_{i}^{e}} R_{i}^{e}+\frac{1}{2} \sum_{e=1}^{N e} \sum_{i=1}^{N r} \sum_{e 2=1}^{N e} \sum_{j=1}^{N r} \frac{\partial^{2}[K]}{\partial R_{i}^{e 1} \partial R_{j}^{e 2}} R_{i}^{e 1} R_{j}^{e 2}+\ldots} \\
& \{U\}\left(R_{1}^{e}, R_{2}^{e}, \ldots\right)=\{U\}_{0}+\sum_{e=1}^{N e} \sum_{i=1}^{N r} \frac{\partial\{U\}}{\partial R_{i}^{e}} R_{i}^{e}+\frac{1}{2} \sum_{e l=1}^{N e} \sum_{i=1}^{N r} \sum_{e 2=1}^{N e} \sum_{j=1}^{N r} \frac{\partial^{2}\{U\}}{\partial R_{i}^{e 1} \partial R_{j}^{e 2}} R_{i}^{e 1} R_{j}^{e 2}+\ldots
\end{aligned}
$$

Substituting Eq. (10) into equilibrium equation:

$$
\begin{aligned}
& {\left[K\left(R_{1}^{e}, R_{2}^{e}, \ldots\right)\right]\left\{U\left(R_{1}^{e}, R_{2}^{e}, \ldots\right)\right\}=\{F\}} \\
& \left\{[K]_{0}+\sum_{e=1}^{N e} \sum_{i=1}^{N r} \frac{\partial[K]}{\partial R_{i}^{e}} R_{i}^{e}+\frac{1}{2} \sum_{e 1=1}^{N e} \sum_{i=1}^{N r} \sum_{e 2=1}^{N e} \sum_{j=1}^{N r} \frac{\partial^{2}[K]}{\partial R_{i}^{e 1} \partial R_{j}^{e 2}} R_{i}^{e 1} R_{j}^{e 2}+\ldots\right\} \\
& \left\{\{U\}_{0}+\sum_{e=1}^{N e} \sum_{i=1}^{N r} \frac{\partial\{U\}}{\partial R_{i}^{e}} R_{i}^{e}+\frac{1}{2} \sum_{e l=1}^{N e} \sum_{i=1}^{N r} \sum_{e 2=1}^{N e} \sum_{j=1}^{N r} \frac{\partial^{2}\{U\}}{\partial R_{i}^{e 1} \partial R_{j}^{e 2}} R_{i}^{e 1} R_{j}^{e 2}+\ldots\right\}=\{F\}
\end{aligned}
$$

We can get the first-order solution from Eq.(11): 
Transport and Communications Science Journal, Vol. 71, Issue 4 (05/2020), 359-367

$$
\begin{aligned}
& {[K]_{0}\{U\}_{0}=\{F\}} \\
& \frac{\partial\{U\}}{\partial R_{i}^{e}}=-[K]_{0}^{-1} \frac{\partial[K]}{\partial R_{i}^{e}}\{U\}_{0}
\end{aligned}
$$

Mean vector and covariance matrix of displacement is computed as follows:

$$
\begin{gathered}
\bar{E}[\{U\}]=\bar{E}\left[\{U\}_{0}+\sum_{e=1}^{N e} \sum_{i=1}^{N r} \frac{\partial\{U\}}{\partial R_{i}^{e}} R_{i}^{e}\right]=[K]_{0}^{-1}\{F\} \\
\operatorname{Cov}[\{U\},\{U\}]=\bar{E}\left[(\{U\}-\bar{E}[\{U\}])^{T}(\{U\}-\bar{E}[\{U\}])\right] \\
=\left\{[K]_{0}^{-1} \frac{\partial[K]}{\partial R_{i}^{e}}\{U\}_{0}\right\}^{T}\left\{[K]_{0}^{-1} \frac{\partial[K]}{\partial R_{j}^{e 2}}\{U\}_{0}\right\} \bar{E}\left[R_{i}^{e 1} R_{j}^{e 2}\right]
\end{gathered}
$$

The coefficient of variation $C O V$ of displacement $U$ defined as follows:

$$
C O V=\frac{\sqrt{\text { Variance }(U)}}{\text { mean }(U)}
$$

\section{NUMERICAL EXAMPLES}

\subsection{Verification example: Bar subjected to triangular distributed load}

Consider a bar problem studied by Rahman [16] with length $L=1$ units, which is subjected to triangular distributed load, $p(x)=x$, in the $x$-direction as shown in Figure 2. The bar has a constant cross-sectional area, $\mathrm{A}=1$ units. The modulus of elasticity, $E(x)$ is random with mean, $E_{0}=1$ units and $r(x)$ is a homogeneous Gaussian random with mean zero and auto-covariance function,

$$
R(\tau)=\sigma_{E}^{2} \exp \left(-\frac{|\tau|}{b L}\right)
$$

where $\sigma_{E}$ is the standard deviation of $r(x)$ or $E(x)$, and $\mathrm{b}$ is the correlation length parameter. For numerical calculations, the following values were used: $\sigma_{E}=1$ and $b=1$.

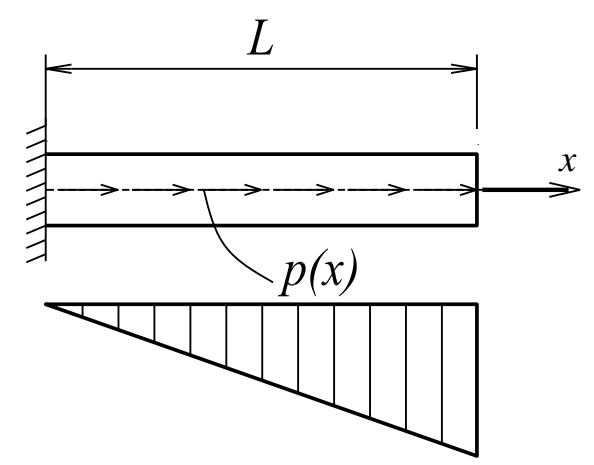

Figure 2. A bar subjected to a triangular distributed load. 
Transport and Communications Science Journal, Vol. 71, Issue 4 (05/2020), 359-367

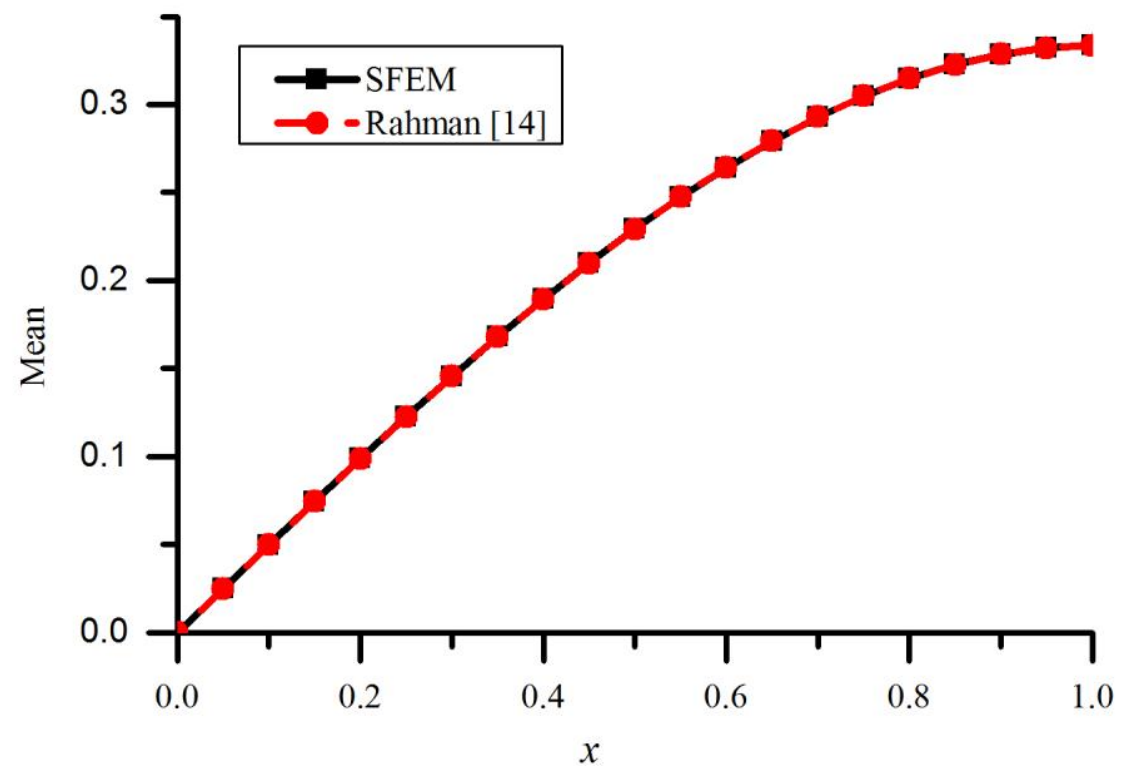

Figure 3. Mean at distance along the bar.

The stochastic finite element method developed in this study was applied to determine the mean and standard deviation of the axial displacement of the bar. Figures 3 and 4 show the mean and standard deviation of the axial displacement predicted by the present approach and stochastic messless method [16]. The stochastic finite element method results agree very well with the stochastic messless method results.

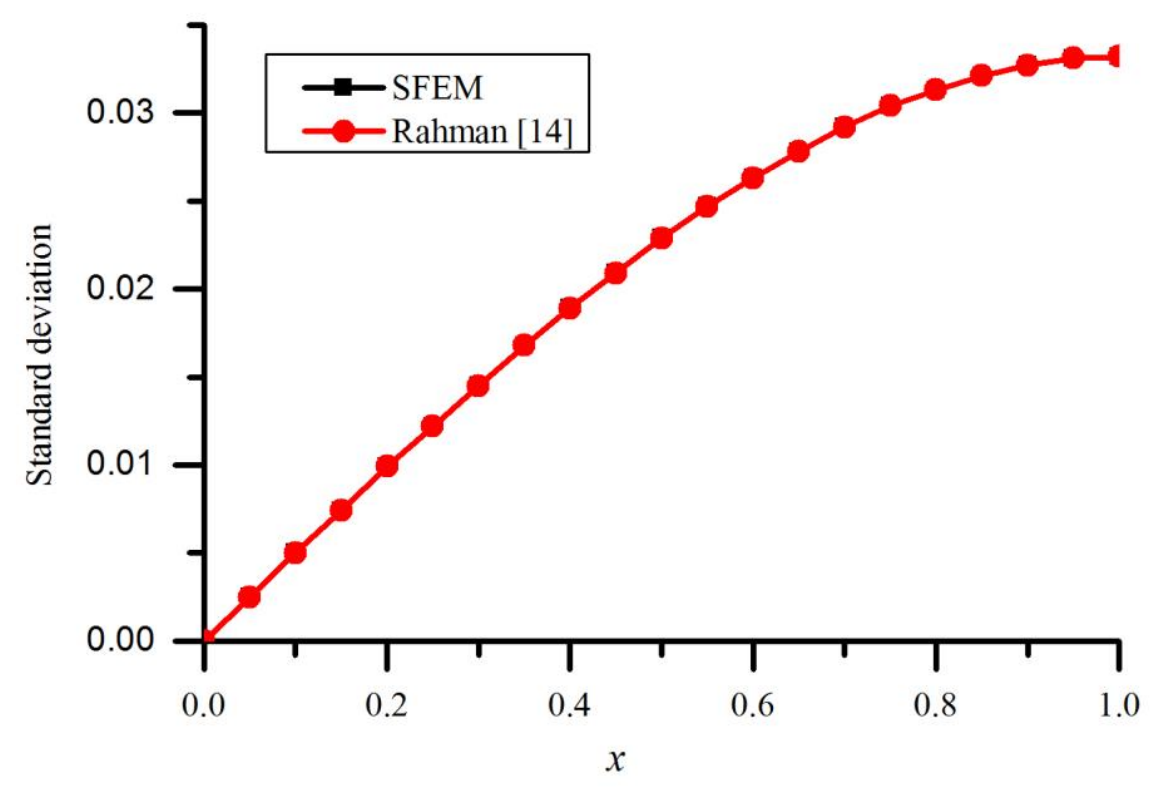

Figure 4. Standard deviation at distance along the bar. 
Transport and Communications Science Journal, Vol. 71, Issue 4 (05/2020), 359-367

\subsection{Nonuniform column example}

A circular concrete column has length $H=10 \mathrm{~m}$, diameters: $1 \mathrm{~m}$ at the bottom and $0,5 \mathrm{~m}$ at the top of the column. A material property of column: the mean of elastic modulus $E_{0}$ $=29 \mathrm{GPa}$, and Poisson's ratio $v=0.3$ and coefficient of variation of a random field of elastic modulus $\sigma=0.1$.

The auto-correlation functions for the respective random field $r(x)$ are assumed as follows:

$$
R(\tau)=\sigma^{2} e^{\left(-\frac{|\tau|}{d}\right)}
$$

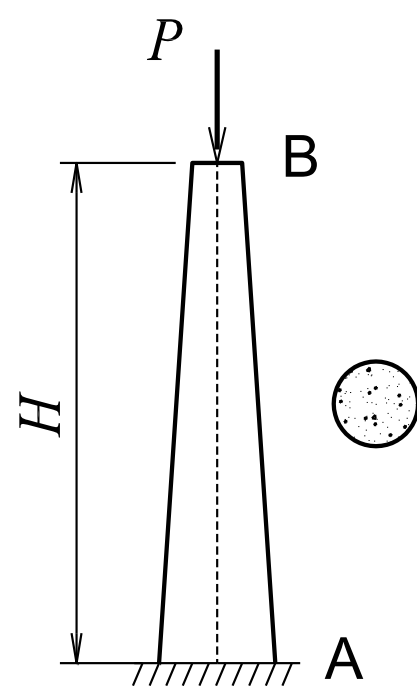

Figure 5. Nonuniform column subjected to a concentrated load at the top.

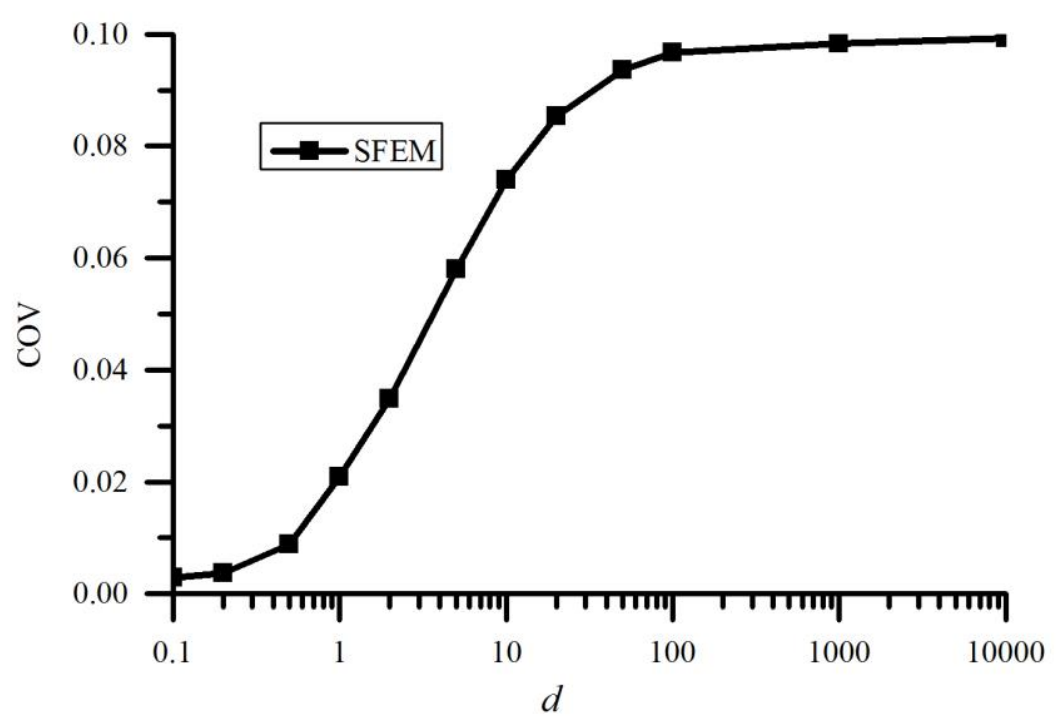

Figure 6. Effect of correlation distance on the coefficient of variation (COV) of displacement at B. 
We have graphed the results of the coefficient of variation COV of displacement by correlation distance of random filed d parameter as shown in figure 6 . The overall behaviors of the COV graph increase from 0.01 to 0.1 . The first one is where length distance parameter $d$ range from 0.1 to 0.5 , in this region, the graph slowly increase from 0.003 to 0.008 . In the last part is from length distance parameter $d$ equal 0.5 to $d$ equals 1000 , in this region, the COV graph increase to the coefficient of variation of random field $E(x)$.

\section{CONCLUSION}

Mean, standard deviation, coefficient of displacements of the nonuniform column are carried out by the stochastic finite element method. A random field of elastic modulus is discretized by a weighted integration technique to formulate a stochastic finite element. Comparing the coefficient of variation of displacement by the present method and previous study show high accuracy if the proposed approach. The effect of length distance parameter $d$ of the random field of elastic modulus on the response COV displacements of the column increase when length distance parameter $d$ goes up. The response COV displacements come to COV of the random field of elastic modulus if the length distance parameter $d$ is over 100 .

\section{ACKNOWLEDGMENT}

This research is funded by the Vietnam National Foundation for Science and Technology Development (NAFOSTED) under grant number 107.01-2017.314.

\section{REFERENCES}

[1]. L. T. Ha, N. T. K. Khue, Free vibration of functionally graded porous nano beams, Transport and Communications Science Journal, 70(2019) 95-103. https://doi.org/10.25073/tcsj.70.2.2

[2]. P. M. Phuc, Analysis free vibration of the functionally grade material cracked plates with varying thickness using the phase-field theory, Transport and Communications Science Journal, 70 (2019) 122-31. https://doi.org/10.25073/tcsj.70.2.5

[3]. G Stefanou, The stochastic finite element method: Past, present and future, Computer Methods in Applied Mechanics and Engineering, 198(2009) 1031-51. http://dx.doi.org/10.1016/j.cma.2008.11.007 [4]. W. K. Liu, T. Belytschko, A.Mani, Random field finite elements, International Journal for Numerical Methods in Engineering, 23(1986) 1831-45. http://dx.doi.org/10.1002/nme.1620231004

[5]. M. Kleiber, T. D. Hien, The stochastic finite element method : basic perturbation technique and $\begin{array}{lll}\text { computer } & \text { implementation: Wiley, } & 1992 .\end{array}$ https://onlinelibrary.wiley.com/doi/abs/10.1002/asm.3150100412

[6]. M. Kaminski, The Stochastic Perturbation Method for Computational Mechanics: WIKEY, 2013. https://www.wiley.com/en-us/The+Stochastic+Perturbation+Method+for+Computational+Mechanicsp-9780470770825

[7]. R. G. Ghanem, P.D.Spanos, Spectral stochastic finite-element formulation for reliability-analysis, Journal of Engineering Mechanics-Asce, 117 (1991) 2351-72. https://doi.org/10.1061/(ASCE)07339399(1991)117:10(2351)

[8]. H. G. Matthies, C. E. Brenner, C. G. Bucher, Guedes Soares C. Uncertainties in probabilistic numerical analysis of structures and solids-Stochastic finite elements, Structural Safety, 19 (1997) 283-336. http://dx.doi.org/10.1016/S0167-4730(97)00013-1

[9]. A. D. Kiureghian, P. L. Liu, Structural Reliability under Incomplete Probability Information, Journal of Engineering Mechanics, 112 (1986) 85-104. https://doi.org/10.1061/(ASCE)0733- 
Transport and Communications Science Journal, Vol. 71, Issue 4 (05/2020), 359-367

9399(1986)112:1(85)

[10].E. Vanmarcke, M. Grigoriu, Stochastic Finite Element Analysis of Simple Beams, Journal of Engineering Mechanics, $109 \quad$ (1983) 1203-14. https://doi.org/10.1061/(ASCE)07339399(1983)109:5(1203)

[11].H-C.Noh, T. Park, Response variability of laminate composite plates due to spatially random material parameter, Computer Methods in Applied Mechanics and Engineering, 200 (2011) 2397-406. http://dx.doi.org/10.1016/j.cma.2011.03.020

[12].H-C Noh, Stochastic finite element analysis of composite plates considering spatial randomness of material properties and their correlations, Steel and Composite Structures, 11 (2011) 115-30. https://doi.org/10.12989/scs.2011.11.2.115

[13].H.C. Noh, Effect of multiple uncertain material properties on the response variability of in-plane and plate structures, Computer Methods in Applied Mechanics and Engineering, 195 (2006) 2697-718. https://doi.org/10.1016/j.cma.2005.06.026

[14].T. D. Hien, N. D. Hung, N. T. Kien, H. C. Noh, The variability of dynamic responses of beams resting on elastic foundation subjected to vehicle with random system parameters, Applied Mathematical Modelling, 67 (2019) 676-87. https://doi.org/10.1016/j.apm.2018.11.018

[15].S. Kitipornchai, J. Yang, K. M. Liew, Random vibration of the functionally graded laminates in thermal environments, Computer Methods in Applied Mechanics and Engineering, 195 (2006) 107595. http://dx.doi.org/10.1016/j.cma.2005.01.016

[16].S. Rahman, B. N. Rao, A perturbation method for stochastic meshless analysis in elastostatics, International Journal for Numerical Methods in Engineering, 50(2001) 1969-1991. https://doi.org/10.1002/nme.106

[17].H. X. Nguyen, T. Duy Hien, J. Lee, H. Nguyen-Xuan, Stochastic buckling behaviour of laminated composite structures with uncertain material properties, Aerospace Science and Technology, 66 (2017) 274-83. https://doi.org/10.1016/j.ast.2017.01.028

[18].T. D. Hien, B. T. Thanh, N.T. Quynh Giang, Uncertainty qualification for the free vibration of a functionally graded material plate with uncertain mass density, IOP Conference Series: Earth and Environmental Science, 143(2018) 012021. http://dx.doi.org/10.1088/1755-1315/143/1/012021

[19].C. Ding, X. Hu, X.Cui, G. Li, Y. Cai, K.K.Tamma, Isogeometric generalized $\mathrm{n}$ th order perturbation-based stochastic method for exact geometric modeling of (composite) structures: Static and dynamic analysis with random material parameters, Computer Methods in Applied Mechanics and Engineering, 346(2019) 1002-1024. https://doi.org/10.1016/j.cma.2018.09.032

[20].T. De Larrard, J. B. Colliat, F. Benboudjema, J. M. Torrenti, G. Nahas, Effect of the Young modulus variability on the mechanical behaviour of a nuclear containment vessel, Nuclear Engineering and Design, 240(2010) 4051-60. https://doi.org/10.1016/j.nucengdes.2010.09.031 